\title{
Elevated neutrophil-to-lymphocyte ratio in the diagnosis of Hashimoto's thyroiditis
}

\author{
Gulali Aktas ${ }^{1 *}$, Mustafa $\mathrm{Sit}^{2}$, Oguz Dikbas ${ }^{3}$, Hayri Erkol $^{2}$, Rabia Altinordu ${ }^{1}$, Edip Erkus ${ }^{1}$, Haluk Savl $^{1}$ \\ ${ }^{1} \mathrm{MD}$, Department of Internal Medicine, Abant Izzet Baysal University Hospital, Bolu, Turkey \\ ${ }^{2} \mathrm{MD}$, Department of General Surgery, Abant Izzet Baysal University Hospital, Bolu, Turkey \\ ${ }^{3} \mathrm{MD}$, Department of Endocrinology, Giresun University Hospital, Giresun, Turkey
}

Study conducted at the Department of Internal Medicine, Abant Izzet Baysal University Hospital, Bolu, Turkey

Article received: $5 / 2 / 2017$ Accepted for publication: 5/22/2017

*Correspondence: Abant Izzet Baysal University Hospital, Department of Internal Medicine Golkoy, Bolu - Turkey Postal code: 14280 draliaktas@yahoo.com

\section{SUMMARY}

Objective: Hashimoto's thyroiditis (HT) is an autoimmune inflammatory disorder. The purpose of this study was to determine the neutrophil-to-lymphocyte ratio (NLR), a novel marker of inflammation, in patients with HT and to compare these values with those from healthy subjects.

Method: A total of 154 participants were included in the study, $90 \mathrm{HT}$ patients and 64 healthy volunteers. Retrospectively, demographic and laboratory data of the subjects were obtained from our institution's database. Patients with active infection, diabetes mellitus, malignancy, other chronic inflammatory diseases, hematologic disorders and patients on aspirin or steroid treatment were excluded from the study. Values for complete blood count (CBC) and serum laboratory parameters of HT patients were the baseline values obtained at the time of HT diagnosis. Control subjects consisted of healthy volunteers who visited our institution for a routine check-up.

Results: Age, gender and CBC parameters were not different between the HT group and the control group; however, the NLR of HT group (2.1 [1.3-5.8]) was significantly higher than the control group (1.9 [0.6-3.3]), $\mathrm{p}=0.04$.

Conclusion: Increased NLR may be useful as an indicator of the presence of HT, especially in complicated cases. NLR is inexpensive and easy to determine. Larger, prospective studies are required to determine its usefulness in assessing diagnostic potential and treatment outcomes in HT patients.

Keywords: Hashimoto disease, blood cell count, inflammation, diagnosis.

\section{INTRODUCTION}

Hashimoto's thyroiditis (HT) is the most common autoimmune thyroiditis worldwide, with prevalence as great as $6 \%$ in iodine-deficient countries. ${ }^{1}$ Typically, the disorder is more frequently seen in women. The characteristic histopathological feature of HT is lymphoplasmacytic inflammation of the thyroid tissue. ${ }^{2}$ Circulating autoantibodies against thyroid peroxidase and thyroglobulin are elevated in the serum of patients with HT.

Complete blood count (CBC)-derived parameters and their relation to certain diseases have recently received attention from researchers. One of these $\mathrm{CBC}$ parameters is the neutrophil-to-lymphocyte ratio (NLR). NLR is considered to be a marker of inflammation and, due to its simplicity and low cost, has been studied in many medi- cal conditions. ${ }^{3}$ An elevated neutrophil count in a CBC predicts ongoing inflammation and decreased lymphocyte count is considered to be an indicator of poor prognosis, so a combination of these two measures is generally accepted to be predictive of an inflammatory situation. ${ }^{3}$ NLR reflects both inflammatory burden (by neutrophil count) and regulatory mechanisms (by lymphocyte count) in inflammatory disease. ${ }^{4,5}$ Studies suggest NLR is associated with occult inflammation in certain conditions. ${ }^{6-8}$ NLR has also been shown to be useful in predicting adverse outcomes in patients with pancreatitis, ${ }^{9}$ appendicitis ${ }^{10}$ and other critical conditions. ${ }^{11}$

NLR has not previously been studied in HT subjects, and, in this retrospective study, NLR was determined in $90 \mathrm{HT}$ subjects and investigated the possible association 
between HT and NLR by comparing these values to NLR determined in a healthy population.

\section{Objective}

Since there is a strong association between inflammation and HT, and between inflammation and NLR, we aimed to compare NLR values of patients with HT to those of healthy volunteers.

\section{Method}

Patients with diagnosis of HT who were followed up in the internal medicine clinics of our institution were enrolled to present retrospective study. Diagnosis of HT was established with a combination of relevant history and findings in physical examination that were supported by characteristic findings on ultrasound scan (diffuse enlargement of the gland and decreased echo pattern) and elevated serum anti-thyroid peroxidase or anti-thyroglobulin levels. Patients with known active infection, diabetes mellitus, malignancy, other chronic inflammatory diseases and hematologic disorders were not included in the study. Patients on steroid or aspirin treatment were also excluded.

$\mathrm{CBC}$ and serum parameters used in this study were the baseline laboratory findings that were recorded in our database at the time of HT diagnosis. Control subjects consisted of healthy volunteers who visited our institution for a routine check-up. General characteristics and laboratory data of all participants were obtained from the computerized database of our clinics. White blood cell count (WBC), neutrophil count (Neu), lymphocyte count (Lym), hemoglobin (Hb), hematocrit (Htc), mean corpuscular volume (MCV) and platelet count (PLT) were recorded for all participants. NLR was calculated simply dividing the Neu value by the Lym value.

The automatic analyzer of the LH 780 Beckman Coulter device (Beckman Coulter Inc.; Brea, CA, USA) was used for blood count analyses in the laboratory of our institution. For laboratory analyses, the original kits of the manufacturer were used. Statistical analysis was conducted using SPSS software (SPSS15.0; SPSS Inc., Chicago, IL, USA). Data were expressed as mean \pm SD or median (min-max). Independent samples t-test or Mann-Whitney $U$ test were used to compare variables in the study population. A p-value lower than 0.05 was considered statistically significant.

\section{RESULTS}

A total of 90 patients with HT and 64 healthy control subjects were enrolled in the study. Mean ages of the HT and control groups were $37 \pm 11$ and $39 \pm 9$ years, respec- tively. The difference was not statistically significant $(p=0.27)$. The HT group consisted of 75 women and 15 men while the control group comprised 56 women and eight men. There was no significant difference between $\mathrm{HT}$ and control groups in terms of gender $(\mathrm{p}=0.47)$. While thyroid stimulating hormone (TSH), free T4 and free T3 levels of all control subjects were normal, 22 of HT subjects were thyrotoxic (suppressed levels of TSH along with elevated T4 or T3). None of the HT patients were hypothyroid. General characteristics and laboratory data of both study populations are shown in Table 1 .

The NLR of HT group (2.1 [1.3-5.8]) was significantly higher than that of the control group $(1.9[0.6-3.3])(\mathrm{p}=0.04)$.

\section{Discussion}

The main finding of our study is that NLR was elevated in HT patients compared with the healthy control subjects. This is the first reported association between HT and NLR.

By 2010, NLR had been introduced as a simple indicator of systemic inflammation in different clinical conditions. ${ }^{12}$ Shimada et al. proposed NLR as a reliable predictor of inflammatory burden. ${ }^{13} \mathrm{C}$-reactive protein (CRP), which responds immediately to infectious or inflammatory stimulus, is one of the most well-established inflammatory markers and, interestingly, NLR was found to correlate with CRP. ${ }^{14,15}$ There are a number of reports studying NLR in various thyroid diseases. Researchers from Taiwan showed that NLR correlated with the size of thyroid tumors. ${ }^{16}$ Moreover, elevated NLR was proposed as a negative prognostic factor for survival in subjects with papillary thyroid cancer. ${ }^{17}$ Aside from thyroid neoplasm, NLR has also been found to correlate with other types of neoplasms. ${ }^{18,19}$ Inflammation plays a critical role in tumor development, progression, clinical presentation and prognosis of cancer. ${ }^{20} \mathrm{HT}$ is also characterized by a prominent inflammatory burden, which is consistent with lymphocytic inflammation of the thyroid gland; ${ }^{21}$ therefore, the increased NLR seen in HT patients compared with controls in this study is likely to be a result of chronic inflammation.

Elevated NLR has been reported in patients with familial Mediterranean fever (FMF) and has emerged as a valuable predictor of the development of amyloidosis. ${ }^{22}$ In addition to inflammation, HT is characterized by autoimmunity, which is defined by reactivity to antigens of thyroidal tissue. ${ }^{23}$ Both thyroid follicular cells and inflammatory cells, involved in HT, are capable of producing cytokines that may exacerbate the autoimmune process and the inflammatory response; ${ }^{24,25}$ therefore, mechanisms similar to those seen in FMF may induce elevated NLR. 
TABLE 1 General characteristics and laboratory data of the study population.

\begin{tabular}{|c|c|c|c|c|}
\hline & & \multicolumn{2}{|l|}{ Group } & \multirow[t]{2}{*}{$\mathbf{P}$} \\
\hline & & HT group & Control group & \\
\hline \multirow[t]{3}{*}{ Gender } & Men (n) & 15 & 8 & \multirow[t]{2}{*}{0.47} \\
\hline & Women (n) & 75 & 56 & \\
\hline & & \multicolumn{2}{|l|}{ Mean \pm SD } & \\
\hline Age (years) & & $37.3 \pm 11.5$ & $39.2 \pm 9$ & 0.27 \\
\hline $\mathrm{WBC}\left(\mathrm{u} / \mathrm{mm}^{3}\right)$ & & $7.5 \pm 1.7$ & $7.1 \pm 1.5$ & 0.66 \\
\hline $\mathrm{Hb}(\mathrm{g} / \mathrm{dL})$ & & $13.6 \pm 1.3$ & $13.6 \pm 1.4$ & 0.15 \\
\hline $\mathrm{Htc}(\%)$ & & $40 \pm 3.5$ & $40.8 \pm 3.8$ & 0.16 \\
\hline $\mathrm{MCV}(\mathrm{fL})$ & & $86 \pm 6$ & $86 \pm 5$ & 0.55 \\
\hline $\operatorname{PLT}\left(\mathrm{u} / \mathrm{mm}^{3}\right)$ & & $299 \pm 63$ & $301 \pm 63$ & 0.91 \\
\hline TSH (ulU/mL) & & $1.2 \pm 1$ & $1.9 \pm 1$ & 0.002 \\
\hline FT4 (ng/dL) & & $1.5 \pm 0.5$ & $1.2 \pm 0.2$ & 0.03 \\
\hline \multirow[t]{2}{*}{ FT3 $(\mathrm{pg} / \mathrm{mL})$} & & $4.3 \pm 0.9$ & $3.3 \pm 0.4$ & 0.03 \\
\hline & & \multicolumn{2}{|c|}{ Median (min-max) } & \\
\hline $\operatorname{Neu}\left(\mathrm{u} / \mathrm{mm}^{3}\right)$ & & $4.3(2.1-9.8)$ & $4.1(2-6.3)$ & 0.36 \\
\hline $\operatorname{Lym}\left(\mathrm{u} / \mathrm{mm}^{3}\right)$ & & $2(0.8-3.5)$ & $2.2(1.3-4.7)$ & 0.08 \\
\hline NLR & & $2.1(1.3-5.8)$ & $1.9(0.6-3.3)$ & 0.04 \\
\hline
\end{tabular}

HT: Hashimoto's thyroiditis; WBC: white blood cell count; Hb: hemoglobin; Htc: hematocrit; MCV: mean corpuscular volume; PLT: platelet count; TSH: thyroid stimulating hormone; FT4: free thyroxine; FT3: free triiodothyronine; Neu: neutrophil count; Lym: lymphocyte count; NLR: neutrophil-to-lymphocyte ratio.

External administration of thyroid stimulating hormone (TSH) stimulates triiodothyronine (T3) production in lymphocytes. ${ }^{26}$ Increased intracellular T3 may stimulate lymphocyte production, causing an elevated lymphocyte count on a CBC. In this study, patients with HT had significantly lower TSH than healthy control subjects. Lower serum TSH might lead to a reduction of intracellular $\mathrm{T} 3$ in lymphocytes, which could result in an increase in NLR. On the other hand, HT should be considered as a situation characterized by inflammatory stress, and $\mathrm{T} 3$ concentration in lymphocytes has been found to decrease under stressful conditions. ${ }^{27}$

In healthy populations, NLR is increased in the elderly; ${ }^{28}$ however, the mean age of the HT patients was not different from the healthy controls in our study so the increase in NLR seen in our study cannot be attributed to this age-related correlation.

Hashimoto's thyroiditis is associated with endothelial dysfunction, ${ }^{29}$ which is considered to be an underlying cause of atherosclerosis and may reflect an early stage in the development of atherosclerosis. ${ }^{30}$ NLR was suggested to be an independent predictor of cardiac mortality in ischemic heart disease. ${ }^{31}$ Moreover, NLR was found to be useful for risk stratification in acute coronary syndrome. ${ }^{8}$ A more recent study revealed that NLR was associated with the severity and the prognosis of ischemic heart disease. ${ }^{32} \mathrm{HT}$ is thought to be associated with endothe- lial dysfunction, and, thus, with atherosclerosis; ${ }^{33}$ therefore, the observation of an elevated NLR in HT, similar to that seen in atherosclerotic diseases, is not surprising.

Although hypothyroidism is more common in HT, initial inflammatory destruction of the gland may cause a transient thyrotoxicosis; therefore, serum T3 and T4 levels were higher in HT patients compared to subjects in present study.

Our study was limited by its retrospective design, which could lead to selection bias, and by the small study cohort that made conclusive interpretation of the results difficult.

\section{Conclusion}

NLR is an inexpensive and easily accessible test and, therefore, we suggest that measurement of NLR may be useful in the diagnosis of HT, especially in complicated cases when a diagnosis is uncertain. Larger prospective studies are required to fully assess the usefulness of NLR to diagnose and assess treatment outcomes in HT patients.

\section{References}

1. Bjoro T, Holmen J, Krüger O, Midthjell K, Hunstad K, Schreiner T, et al. Prevalence of thyroid disease, thyroid dysfunction and thyroid peroxidase antibodies in a large, unselected population. The Health Study of NordTrondelag (HUNT). Eur J Endocrinol. 2000; 143(5):639-47.

2. Tunbridge WM, Brewis M, French JM, Appleton D, Bird T, Clark F, et al. Natural history of autoimmune thyroiditis. Brit Med J (Clin Res Ed). 1981; 282(6260):258-62. 
3. Imtiaz F, Shafique K, Mirza SS, Ayoob Z, Vart P, Rao S. Neutrophil lymphocyte ratio as a measure of systemic inflammation in prevalent chronic diseases in Asian population. Int Arch Med. 2012; 5(1):2.

4. Avanzas P, Quiles J, López de Sá E, Sánchez A, Rubio R, García E, et al. Neutrophil count and infarct size in patients with acute myocardial infarction. Int J Cardiol. 2004; 97(1):155-6.

5. Ommen SR, Hodge DO, Rodeheffer RJ, McGregor CG, Thomson SP, Gibbons RJ. Predictive power of the relative lymphocyte concentration in patients with advanced heart failure. Circulation. 1998; 97(1):19-22.

6. Cho H, Hur HW, Kim SW, Kim SH, Kim JH, Kim YT, et al. Pre-treatment neutrophil to lymphocyte ratio is elevated in epithelial ovarian cancer and predicts survival after treatment. Cancer Immunol Immunother. 2009; 58(1):15-23.

7. Gomez D, Morris-Stiff G, Toogood GJ, Lodge JP, Prasad KR. Impact of systemic inflammation on outcome following resection for intrahepatic cholangiocarcinoma. J Surg Oncol. 2008; 97(6):513-8.

8. Tamhane UU, Aneja S, Montgomery D, Rogers EK, Eagle KA, Gurm HS. Association between admission neutrophil to lymphocyte ratio and outcomes in patients with acute coronary syndrome. Am J Cardiol. 2008; 102(6):653-7.

9. Azab B, Jaglall N, Atallah JP, Lamet A, Raja-Surya V, Farah B, et al. Neutrophillymphocyte ratio as a predictor of adverse outcomes of acute pancreatitis. Pancreatology. 2011; 11(4):445-52

10. Goodman DA, Goodman CB, Monk JS. Use of the neutrophil:lymphocyte ratio in the diagnosis of appendicitis. Am Surg. 1995; 61(3):257-9.

11. Zahorec R. Ratio of neutrophil to lymphocyte counts: rapid and simple parameter of systemic inflammation and stress in critically ill. Bratisl Lek Listy. 2001; 102(1):5-14

12. Moore MM, Chua W, Charles KA, Clarke SJ. Inflammation and cancer: causes and consequences. Clin Pharmacol Ther. 2010; 87(4):504-8.

13. Shimada H, Takiguchi N, Kainuma O, Soda H, Ikeda A, Cho A, et al. High preoperative neutrophil-lymphocyte ratio predicts poor survival in patients with gastric cancer. Gastric Cancer. 2010; 13(3):170-6.

14. Kao SC, Pavlakis N, Harvie R, Vardy JL, Boyer MJ, van Zandwijk N, et al. High blood neutrophil-to-lymphocyte ratio is an indicator of poor prognosis in malignant mesothelioma patients undergoing systemic therapy. Clin Cancer Res. 2010; 16(23):5805-13

15. Ohno Y, Nakashima J, Ohori M, Hatano T, Tachibana M. Pretreatment neutrophil-to-lymphocyte ratio as an independent predictor of recurrence in patients with nonmetastatic renal cell carcinoma. J Urol. 2010; 184(3):873-8.

16. Liu CL, Lee JJ, Liu TP, Chang YC, Hsu YC, Cheng SP. Blood neutrophil-tolymphocyte ratio correlates with tumor size in patients with differentiated thyroid cancer. J Surg Oncol. 2013; 107(5):493-7.

17. Kim JY, Park T, Jeong SH, Jeong CY, Ju YT, Lee YJ, et al. Prognostic importance of baseline neutrophil to lymphocyte ratio in patients with advanced papillary thyroid carcinomas. Endocrine. 2014; 46(3):526-31.

18. Bruckner HW, Lavin PT, Plaxe SC, Storch JA, Livstone EM. Absolute granulocyte, lymphocyte, and moncyte counts. Useful determinants of prognosis for patients with metastatic cancer of the stomach. JAMA. 1982; 247(7):1004-6.

19. Satomi A, Murakami S, Ishida K, Mastuki M, Hashimoto T, Sonoda M Significance of increased neutrophils in patients with advanced colorectal cancer. Acta Oncol. 1995; 34(1):69-73.

20. Mantovani A, Allavena P, Sica A, Balkwill F. Cancer-related inflammation. Nature. 2008; 454(7203):436-44

21. Hammerstad SS, Jahnsen FL, Tauriainen S, Hyöty H, Paulsen T, Norheim I, et al. Inflammation and increased myxovirus resistance protein A expression in thyroid tissue in the early stages of Hashimoto's thyroiditis. Thyroid. 2013; 23(3):334-41.

22. Uslu AU, Deveci K, Korkmaz S, Aydin B, Senel S, Sancakdar E, et al. Is neutrophil/lymphocyte ratio associated with subclinical inflammation and amyloidosis in patients with familial Mediterranean fever? Biomed Res Int. $2013 ; 2013: 185317$

23. Weetman AP. Autoimmune thyroid disease. Autoimmunity. 2004;37(4):337-40

24. García-López MA, Sancho D, Sánchez-Madrid F, Marazuela M. Thyrocytes from autoimmune thyroid disorders produce the chemokines IP-10 and Mig and attract CXCR3+ lymphocytes. J Clin Endocrinol Metab. 2001; 86(10):5008-16.

25. Ajjan RA, Weetman AP. Cytokines in thyroid autoimmunity. Autoimmunity 2003; 36(6-7):351-9.

26. Csaba G, Pállinger E. Thyrotropic hormone (TSH) regulation of triiodothyronine (T(3)) concentration in immune cells. Inflamm Res. 2009; 58(3):151-4.

27. Csaba G, Kovács P, Tóthfalusi L, Pállinger E. Prolonged effect of stress (water and food deprivation) at weaning or in adult age on the triiodothyronine and histamine content of immune cells. Horm Metab Res. 2005; 37(11):711-5.

28. Li J, Chen QY, Luo XH, Hong J, Pan K, Lin X, et al. Neutrophil-to-lymphocyte ratio positively correlates to age in healthy population. J Clin Lab Anal. 2015; 29(6):437-43

29. Taddei S, Caraccio N, Virdis A, Dardano A, Versari D, Ghiadoni L, et al. Lowgrade systemic inflammation causes endothelial dysfunction in patients with Hashimoto's thyroiditis. J Clin Endocrinol Metab. 2006; 91(12):5076-82.

30. Ross R. The pathogenesis of atherosclerosis--an update. N Engl J Med. 1986; 314(8):488-500.

31. Papa A, Emdin M, Passino C, Michelassi C, Battaglia D, Cocci F. Predictive value of elevated neutrophil-lymphocyte ratio on cardiac mortality in patients with stable coronary artery disease. Clin Chim Acta. 2008; 395(1-2):27-31.

32. Arbel Y, Finkelstein A, Halkin A, Birati EY, Revivo M, Zuzut M, et al Neutrophil/lymphocyte ratio is related to the severity of coronary artery disease and clinical outcome in patients undergoing angiography. Atherosclerosis. 2012; 225(2):456-60

33. Xiang GD, He YS, Zhao LS, Hou J, Yue L, Xiang HJ. Impairment of endothelium-dependent arterial dilation in Hashimoto's thyroiditis patients with euthyroidism. Clin Endocrinol. 2006; 64(6):698-702. 